\title{
METODOLOGÍA APLICADA EN EL ESTUDIO DEL DESARROLLO DEL LENGUAJE EN NIÑOS CON DETECCIÓN TEMPRANA DE LA HIPOACUSIA NEONATAL
}

\section{Methodology applied in the study of the language development in children with early detection of neonatal hearing loss}

José Ignacio BENITO-OREJAS; Rosa Belén SANTIAGO-PARDO; Carmen ROMERO-UREÑA; Ángel Luis SÁNCHEZ-ROSSO; María Fe MUÑOZ-MORENO; Marina ESCARDA-BOLAÑOS; Ana HERRERO-GALIACHO; Celia GONZÁLEZ-NÚÑEZ; María Isabel Rico-Paíno; Rebeca Álvarez-Mielgo; Lidia MORENO-FERNÁNDEZ; María CONDE-GONZÁLEZ; Miriam GONZÁLEZ-DEL DEDO; Jorge GUTIÉRREZ-BLANCO; Cristina ARROYO-FERNÁNDEZ; Rocío CERREJÓNMARTÍN; ALBERTO MARTÍN-ROMÁN; Lucía Isabel de CASTRO-DÍEZ; Aarón GARCÍACABALLERO; María FERNÁNDEZ-GÓMEZ; Cristina GARCÍA-ÁLVAREZ

SACYL y Universidad de Valladolid. Hospital Clínico Universitario de Valladolid. Servicio de Otorrinolaringología y PCF. Facultad de Educación (Departamento de Pedagogía) y Facultad de Medicina (Grado en Logopedia) de la Universidad de Valladolid. Grupo SERENDIPIA de Audición y Lenguaje. Valladolid. España.

Correspondencia: jibenito@ono.com

Fecha de recepción: 8 de octubre de 2016

Fecha de aceptación: 13 de noviembre de 2016

Fecha de publicación: 21 de noviembre de 2016

Fecha de publicación del fascículo: 1 de junio de 2017

Conflicto de intereses: Los autores declaran no tener conflictos de intereses

Imágenes: Los autores declaran haber obtenido las imágenes con el permiso de los pacientes

Política de derechos y autoarchivo: se permite el autoarchivo de la versión post-print (SHERPA/RoMEO)

Licencia CC BY-NC-ND. Licencia Creative Commons Atribución-NoComercial-SinDerivar 4.0 Internacional

๔) Universidad de Salamanca. Su comercialización está sujeta al permiso del editor neonatal disminuye con una detección e intervención tempranas, hemos elaborado un proyecto de investigación, con el que nos proponemos conocer el grado de desarrollo del lenguaje de los niños y niñas que procedentes del cribado auditivo universal, han sido diagnosticados/as por nosotros de hipoacusia prelingual en estos últimos 15 años y analizar las variables determinantes y las que son modificables. El objeto de esta comunicación consistirá en presentar la metodología que vamos a utilizar. Método: Partimos de los datos almacenados en nuestro Servicio de ORL, que comprende a 282 niños con hipoacusia. Hemos tenido la oportunidad de crear un grupo de investigación en el que coincidimos especialistas de la audición infantil y del lenguaje, por lo que contamos con medios suficientes para el estudio. Resultados: Describimos los elementos que configuran este proyecto, en relación al equipo de trabajo y a su desarrollo. Tras aplicar unos criterios de exclusión/inclusión, hemos seleccionado a un grupo de 45 niños entre 3 y 15 años, definiendo sus características auditivas. Mediante pruebas específicas, adaptadas a la edad, estudiaremos los diferentes aspectos del lenguaje; y a través 
de una entrevista estructurada realizada a los padres, intentaremos determinar las variables que influyen en el proceso re-habilitador. Finalmente, los datos serán analizados estadísticamente. Discusión: La variabilidad y la escasa prevalencia de la hipoacusia infantil, dificultan la realización de estudios con población suficiente para obtener resultados estadísticamente significativos. Sin embargo, creemos que el grupo de niños seleccionado y la metodología utilizada nos permitirán conocer mejor las variables influyentes en el desarrollo del lenguaje. Conclusiones: El programa de cribado auditivo universal ha permitido una intervención más precoz, lo que debería mejorar los niveles de lenguaje de los niños detectados/as con hipoacusia. Aunque el desarrollo normalizado de la comunicación depende de otros factores difíciles de determinar, a través del protocolo presentado pretendemos equiparar estos resultados, validando el proceso de cribado/diagnóstico e intervención de nuestro medio.

PALABRAS CLAVE niño; pérdida auditiva; lenguaje infantil; diagnóstico; habla; sordera

SUMMARY

Introduction and objective: Given that the disabling potential causing neonatal hearing impairment decreases with early detection and intervention, we have drawn up a research project, with which we intend to know the degree of development of the language of children and girls than from the universal hearing screening, have been diagnosed for us of prelingual hearing loss in the last 15 years and analyze the determining variables and which are modifiable. The object of this communication will be to present the methodology that we use. Method: We assume the data stored on our ENT service, including 282 children with hearing loss. We have had the opportunity to create a research group in which we agree ENT and language specialists so we have resources sufficient for the study. Results: We describe the elements that make up this project in relation to the team and to its development. After applying inclusion/exclusion criteria, we have selected a group of 45 children between 3 and 15 years, defining their auditory characteristics. Through specific tests, adapted to the age, we will study the different aspects of the language; and through a structured interview parents, we try to determine the variables that influence the rehabilitator process. Finally, the data will be analyzed statistically. Discussion: The variability and the low prevalence of infant hearing loss, make it difficult studies with sufficient population to obtain statistically significant results. However, we believe that the group of children and the methodology selected will allow us to learn more about the influential in the development of the language variables. Conclusions: The hearing screening program allowed us to earlier intervention, which should improve the levels of language of children detected with hearing loss. Although the standard development of communication depends on other factors difficult to determine, through the presented protocol we equate these findings, validating the process of screening/diagnosis and intervention of our environment.

KEYWORDS

child; hearing loss; child language; diagnosis; speech; deafness

\section{INTRODUCCIÓN}

En las sociedades desarrolladas, la hipoacusia congénita es el desorden sensorial más frecuente [1], afectando a un $3 \%$ de recién nacidos (RN) [2]. Aproximadamente el $95 \%$ de estos niños tienen a uno de sus padres oyente [3] $y$, por tanto, la comunicación oral es una meta comprensible y deseable para estas familias [4]. Pero no debemos olvidar que la sordera no solo impacta negativamente en el desarrollo del lenguaje, sino que influye también en las funciones mentales, en los logros educativos, en el comportamiento social y en las oportunidades socioeconómicas y de empleo laboral [5], suponiendo un importante coste a la sociedad por los cuidados que precia y por la pérdida de productividad que genera [6].

Una manera de disminuir el impacto de la hipoacusia infantil consiste en identificar a los afectados/as, tan pronto como sea posible, por medio de un programa de cribado auditivo neonatal. La posibilidad de poder conocer la audición del recién nacido, ha sido desde siempre el deseo de los profesionales que trabajaban en la habilitación de la sordera, convencidos de que a través de una detección e intervención tempranas se mejorarían las expectativas comunicativas del paciente [7]. Y esta hipótesis se ha hecho actualmente realidad, merced a los últimos avances tecnológicos y a un mayor conocimiento de la función auditiva, lo que ha permitido elaborar nuevas estrategias de diagnóstico precoz y comprobar científicamente que el potencial discapacitante de esta enfermedad, disminuye en gran medida con la intervención temprana [8-10]. Pero, además, los datos acumulados demuestran que su aplicación a todos los RN («universal») se justifica incluso desde el punto de vista económico [11], por lo que la elaboración de «Programas de Cribado de Hipoacusia Neonatal» se ha convertido en todo el mundo desarrollado, en una prioridad dentro de la sanidad pública [12]. En España, el 26 de marzo de 1999 se aprobó en el Congreso de los Diputados una proposición no de ley que instaba al gobierno a articular un 
«Plan Nacional de Prevención de la Sordera Infantil», que se puso en marcha a través de la CODEPEH -Comisión para la Detección Precoz de Hipoacusia-, integrada por miembros de las sociedades de Otorrinolaringología, Pediatría, asociación de padres FIAPAS y Ministerio de Sanidad [13]. En el año 2001 iniciamos en el Hospital Clínico Universitario de Valladolid (HCU-VA) la aplicación de un protocolo de cribado auditivo de todos los recién nacidos [14], que se instauró en el resto de hospitales de la comunidad de Castilla y León en el año 2004 [15].

Dado que el cribado auditivo neonatal permite la identificación temprana de la hipoacusia y el rápido acceso a un tratamiento precoz aprovechando la máxima plasticidad de las funciones cerebrales, queremos conocer cuál ha sido el resultado de este proceso tras su aplicación durante los últimos 15 años. El lenguaje representa la premisa fundamental en la justificación de estos programas, al establecerse que su alteración depende de la severidad de la pérdida auditiva, así que nos planteamos un proyecto que nos permitiera analizar los principales aspectos del lenguaje, en los niños y niñas diagnosticados/as de hipoacusia permanente en el HCU-VA. En este trabajo pretendemos presentar la metodología utilizada para conseguir dicho objetivo.

\section{MATERIAL Y MÉTODO}

1. EQUIPO DE TRABAJO

La coordinación del estudio se realiza por cuatro personas expertas en diferentes materias. Un médico especialista en ORL, responsable del cribado auditivo y del control y seguimiento de la hipoacusia infantil en el servicio de ORL del HCU-VA, dos doctoras en Pedagogía especializadas en discapacidad auditiva, logopedas y profesoras del departamento de Pedagogía, -una de ellas ejerciendo en la inspección educativa y otra conocedora de la lengua de signos-, y finalmente, un profesor de Psicología, competente en las distintas pruebas de lenguaje. Estos tres profesores imparten docencia en las facultades de Educación y de Medicina (Grado en Logopedia) de la Universidad de Valladolid (UVa).

Se cuenta además, con un equipo de dieciséis participantes, logopedas y estudiantes del Grado en Logopedia, que en cuatro grupos de trabajo serán los encargados de recopilar la información y categorizar los resultados. Cada grupo está formado por cuatro personas, tres estudiantes de $3^{\circ}$ y $4^{\circ}$ curso del Grado en Logopedia tutelados por una logopeda.

Finalmente, una persona experta en bioestadística de la Unidad de Apoyo a la Investigación del HCU-VA, será la encargada de realizar el estudio estadístico.

El intercambio de información entre tantos participantes se consigue, en tiempo real, a través de una plataforma de teledocencia (Moodle) instalada en el Campus Virtual de la UVa. En dicha plataforma se abren, a disposición de todos los miembros del proyecto, diferentes foros donde se debaten temas, se insertan calendarios de tareas, se convocan reuniones y se comparten los distintos documentos con los que se trabaja. El análisis final de los casos se realiza a través de sesiones clínicas presenciales.

\section{SUJETOS DE ESTUDIO}

CRITERIOS DE INCLUSIÓN E IDENTIFICACIÓN DEL CASO: los niños objeto de estudio serán aquellos con hipoacusia neonatal o prelingual bilateral, que hayan sido evaluados en estos últimos 15 años en el HCU-VA, con edades comprendidas entre los 3 y los 15 años y sin criterios de exclusión. Su selección se realizará a partir de la base de datos del servicio ORL, donde se encuentran registrados todos los niños con hipoacusia, diagnosticados en el HCU-VA desde 1991.

Por regla general, mantenemos el criterio universal «plan 1-3-6» [1, 13] que significa, cribado auditivo durante el primer mes de vida con potenciales evocados auditivos del tronco cerebral automatizados (PEATC-A), de mayor eficacia para nosotros que las otoemisiones acústicas (OEA) [16, 17]; diagnóstico en los tres primeros meses e inicio de la intervención antes del sexto mes [2, 14, 15, 18]. El diagnóstico audiológico de los niños que "no pasan» el cribado auditivo universal (procedentes de nuestro hospital o de otro centro), se alcanza tras una cuidadosa otomicroscopía e impedanciometría 226/1000 Hz, mediante la aplicación de pruebas auditivas electrofisiológicas: OEA (transitorias y productos de distorsión), PEATC, potenciales evocados de estado estable y a partir de los 8 meses de edad a través de la audiometría del comportamiento con refuerzo visual, suplementada en edades posteriores con la audiometría condicionada operante infantil, audiometría verbal y audiometría de juego. Una vez establecido el diagnóstico de la pérdida auditiva, las revisiones audiológicas se realizan cada 6 meses. 
Para clasificar el grado de hipoacusia, se obtiene la media de las frecuencias $0,5,1,2$ y 4 $\mathrm{kHz}$ en $\mathrm{dB} \mathrm{HL}$ de la prueba auditiva más reciente (audiometría tonal, con refuerzo visual o PEATC) y se aplica la clasificación de la European Bureau International d'Audio-Phonologie (BIAP) (http://www.biap.org/en/recommendations/65-ct-2-classification-des-surdites/5-recommendation-biap-021-bis). La pérdida auditiva en función del oído mejor, puede ser leve (30 a $40 \mathrm{~dB})$, moderada (41 a $70 \mathrm{~dB}$ ), severa (71 a $90 \mathrm{~dB}$ ) o profunda (> $90 \mathrm{~dB}$ ), unilateral o bilateral.

En general, sólo estudiaremos a aquellos niños, cuya pérdida auditiva en el mejor oído, sea de carácter permanente y mayor de $20 \mathrm{~dB}$ $\mathrm{HL}$, de cualquier tipo, salvo de causa central y originada fundamentalmente antes de los 2 años de edad.

CRITERIOS DE EXCLUSIÓN: importante discapacidad intelectual. Hipoacusia unilateral. Hipoacusia progresiva. Los que en el momento del estudio presenten audición normal. No tener como primera lengua el español. Rechazo a la realización de las pruebas. Datos incompletos o no válidos. Falta de seguimiento y/o fallecimiento.

\section{DISEÑO DEL TRABAJO}

Citamos a las familias de los niños seleccionados para explicarles el proyecto y requerir su consentimiento firmado. Tras la aceptación, les entregamos un cuestionario escrito con varias de las preguntas que constan en la entrevista oral que se les realizará posteriormente, a fin de que puedan contestarlas tranquilamente en su domicilio. Aprovechamos el encuentro personal para pedirles todos los informes logopédicos y psicopedagógicos que tengan o puedan solicitar en su colegio o centro al que ha asistido o en el que está siendo atendido su hijo o hija. Procuramos recoger y analizar esta información antes de la siguiente cita de la familia con los entrevistadores.

Así pues, antes de la siguiente cita, para consensuar entre todos los participantes del proyecto el formato idóneo de la entrevista y el pase de las pruebas, se realiza una jornada de simulación, ensayando la mejor manera de que éstos interactúen con el niño durante la aplicación de las pruebas del lenguaje y el procedimiento a seguir para la utilización del video/audio. Como resultado final de la jornada, se elabora un protocolo de actuación para los grupos de trabajo, estableciendo unas normas generales de actitud y comportamiento, que sirvan de guía en el trato interpersonal y en la ejecución de la exploración.

Tras la firma de los consentimientos informados por parte de padres/tutores (se les entrega una copia con posibilidad de revocación), cada grupo cita a las familias seleccionadas en la Facultad de Medicina, donde se habilitan estancias confortables y silenciosas para los padres y adaptadas a un ambiente infantil (mobiliario, material lúdico, estimulante y didáctico...) para los niños. Aunque el tiempo dedicado a cada caso será variable en función de su edad y características, se calculan unas dos sesiones, de aproximadamente una hora y media de duración máxima cada una, para niños pequeños y una única sesión de hasta dos horas para los más mayores. En todo momento y velando por el buen hacer, los coordinadores del trabajo supervisan y apoyan todas estas actuaciones.

Con toda la información recabada de cada uno de los casos, se abre un dossier individual: consentimiento informado, cuestionario cumplimentado, informes audiológicos, informes logopédicos e informes psicopedagógicos aportados por los padres.

La intervención a realizar constará de dos partes, que serán llevadas a cabo simultáneamente en dependencias separadas; una entrevista a los padres y la realización de las pruebas específicas del lenguaje al niño:

-ENTREVISTA A PADRES/TUTORES: donde se recogerá información de los padres o cuidadores de aquellas variables que pudieran influir en la evolución del lenguaje. Previamente a este encuentro se han revisado tanto el cuestionario escrito entregado, tras la firma del consentimiento informado, como los informes logopédicos y psicopedagógicos aportados por la familia, por lo que la entrevista va dirigida a matizar las respuestas y a completar los datos que falten.

PRUebAs de LenguAJE: para evaluar la competencia lingüística de los casos se aplicarán diferentes pruebas en función de la edad. Es importante que durante su realización los niños lleven colocados sus audífonos o su implante coclear (IC). El desarrollo de cada prueba se acomete de acuerdo al procedimiento descrito en la propia prueba.

No se suministra toda la batería seleccionada en este proyecto para la evaluación del lenguaje a cada niño, sino en función de sus características personales, de los resultados que 
se vayan obteniendo y de las pruebas que previamente ya les hayan pasado.

\section{EVALUACIÓN DE RESULTADOS}

Los datos obtenidos, relativos a la caracterización de la pérdida auditiva, a las variables incluidas en la entrevista y al resultado de las pruebas de lenguaje, serán introducidos en una base de datos Microsoft Access elaborada exprofeso, para su explotación estadística. Las variables cuantitativas se presentarán con la media y la desviación típica para aquellas con distribución normal y con la mediana y la amplitud intercuartil en las que no sigan esta distribución, y las cualitativas según su configuración de frecuencias. Mediante el test Chicuadrado de Pearson, se analizará la asociación de las variables cualitativas. En el caso de que el número de celdas con valores esperados menores de 5 sea mayor de un $20 \%$, se utilizará el test exacto de Fisher o el test Razón de Verosimilitud para variables con más de dos categorías. Las comparaciones de los valores cuantitativos se realizarán mediante la prueba $T$ de Student o el ANOVA de un factor para muestras independientes según si el número de grupos a comparar es dos o mayor. Aplicaremos un análisis de regresión logística multivariante para identificar los factores de riesgo asociados con el desarrollo del lenguaje, incluyéndose las variables que resulten ser estadísticamente significativas en los análisis previos. Los datos serán analizados con el programa estadístico IBM SPSS Statistics versión 20.0 para Windows. Aquellos valores de $p<0,05$ serán considerados estadísticamente significativos.

\section{RESULTADOS}

Este trabajo ha sido aprobado por el Comité Ético de Investigación Clínica del Área de Salud de Valladolid, al que pertenece el HCU-VA (CEIC-VA-ESTE-HCUV).

Todos los miembros del equipo han firmado un compromiso de participación y una declaración específica de confidencialidad y secreto profesional (documento interno de declaración de confidencialidad y secreto profesional).

El consentimiento informado entregado a las familias, ha recibido la aprobación del CEIC (documento interno de consentimiento informado para investigación clínica que no implique muestras biológicas) pudiendo ser revocado en cualquier momento.
Los niños objeto de estudio son aquellos diagnosticados en el HCU-VA desde el año 2001, en que comenzamos el cribado auditivo universal [14]. Contamos con una base de datos de 282 niños y niñas con hipoacusia, que incluye a los que proceden del cribado auditivo del hospital y a todos los que fueron remitidos durante este tiempo, para estudio diagnóstico. La evaluación lingüística realizada abarca a los niños con edades comprendidas entre los 3 años (edad mínima que permite realizar una exploración formal del lenguaje) y los 15 años. Por tanto, la población de estudio son los niños registrados/as, cuyo nacimiento esté comprendido entre el 01-06-2001 (15 años) y 01-062013 (3 años), lo que corresponde a 144 de los 282. Además, salvo excepciones, hemos tenido en cuenta los siguientes parámetros para la selección (Tabla 1): vivir en Valladolid ciudad o provincia; ser la hipoacusia permanente y bilateral, con una pérdida en el mejor oído mayor de $20 \mathrm{~dB}$ HL (se descartan las hipoacusias unilaterales), de cualquier tipo (salvo hipoacusias centrales); no presentar un retraso intelectual severo; haber realizado un seguimiento apropiado (por este motivo, se excluye a gran parte de los sujetos de etnia gitana) y finalmente, diagnosticados con una edad inferior a 1 año. Pero hemos incluido a 17 niños que no cumplía alguna/as de estas condiciones, con fines comparativos. El número de casos inicialmente seleccionado ha sido de 45 .

Tabla 1. Criterios de selección de la población de estudio.

\begin{tabular}{|l|c|c|l|}
\hline \multicolumn{1}{|c|}{ VARIABLES } & $\mathrm{N}$ & Resto & \multicolumn{1}{|c|}{ EXCEPCIONES } \\
\hline $\begin{array}{l}\text { POBLACIÓN INICIAL } \\
\text { BASE DE DATOS: }\end{array}$ & 282 & & \\
\hline Entre 3 y 15 años: & 144 & 138 & NO \\
\hline $\begin{array}{l}\text { Valladolid ciudad y } \\
\text { provincia: }\end{array}$ & 124 & 20 & 1 de Palencia \\
\hline $\begin{array}{l}\text { Descartamos hipo uni- } \\
\text { laterales: }\end{array}$ & 97 & 27 & NO \\
\hline $\begin{array}{l}\text { Descartamos retraso } \\
\text { psiquico severo: }\end{array}$ & 72 & 25 & $\begin{array}{l}7 \text { con retraso mode- } \\
\text { rado }\end{array}$ \\
\hline $\begin{array}{l}\text { Descartamos etnia gi- } \\
\text { tana: }\end{array}$ & 58 & 14 & 3 de etnia gitana \\
\hline $\begin{array}{l}\text { Descartamos falta de } \\
\text { seguimiento: }\end{array}$ & 47 & 11 & $\begin{array}{l}\text { 8 con seguimiento } \\
\text { discontinuo }\end{array}$ \\
\hline $\begin{array}{l}\text { Diagnosticados en el } \\
\text { primer año: }\end{array}$ & 28 & 19 & $\begin{array}{l}13 \text { con diagnóstico > } \\
1 \text { año (por hipoacusia } \\
\text { tardia o desconocida) }\end{array}$ \\
\hline $\begin{array}{l}\text { POBLACIÓN FINAL } \\
\text { SELECCIONADA: }\end{array}$ & 45 & & $\begin{array}{l}\text { Se incluyen excep- } \\
\text { ciones, donde algu- } \\
\text { nos pacientes com- } \\
\text { parten varias. }\end{array}$ \\
\hline
\end{tabular}


DESARROLLO DEL LENGUAJE EN NIÑOS CON DETECCIÓN TEMPRANA DE LA HIPOACUSIA NEONATAL BENITO-OREJAS JI ET AL.

Tabla 2. Características generales y auditivas de los pacientes seleccionados.

\begin{tabular}{|c|c|c|}
\hline Variable & $\mathrm{n}$ & $\%$ \\
\hline POBLACIÓN DE ESTUDIO: & 45 & \\
\hline \multicolumn{3}{|l|}{ GÉNERO: } \\
\hline - Varones & 32 & $71 \%$ \\
\hline Hembras & 13 & $29 \%$ \\
\hline \multicolumn{3}{|l|}{ DISTRIBUCIÓN POR EDADES: } \\
\hline - 3 años & 1 & $2 \%$ \\
\hline 4 años & 4 & $9 \%$ \\
\hline 5 años & 3 & $7 \%$ \\
\hline 6 años & 5 & $11 \%$ \\
\hline 7 años & 1 & $2 \%$ \\
\hline 8 años & 4 & $9 \%$ \\
\hline 9 años & 6 & $13 \%$ \\
\hline 10 años & 6 & $13 \%$ \\
\hline 11 años & 3 & $7 \%$ \\
\hline 12 años & 3 & $7 \%$ \\
\hline 13 años & 1 & $2 \%$ \\
\hline 14 años & 4 & $9 \%$ \\
\hline - 15 años & 4 & $9 \%$ \\
\hline ETNIA GITANA: & 3 & $7 \%$ \\
\hline RETRASO PSICOMOTOR: & 7 & $15 \%$ \\
\hline CON FACTORES DE RIESGO: & 32 & $71 \%$ \\
\hline \multicolumn{3}{|l|}{ EDAD DE DIAGNÓSTICO: } \\
\hline - $\quad$ Con $<1$ año & 32 & $71 \%$ \\
\hline Entre 1 y 2 años & 5 & $11 \%$ \\
\hline - $\quad$ Con $>2$ años & 8 & $18 \%$ \\
\hline \multicolumn{3}{|l|}{ PROCEDENCIA: } \\
\hline - $\quad$ Cribado universal HCU & 18 & $40 \%$ \\
\hline - $\quad$ Otra procedencia & 27 & $60 \%$ \\
\hline \multicolumn{3}{|l|}{ LUGAR DE ORIGEN: } \\
\hline - $\quad$ Valladolid ciudad & 32 & $71 \%$ \\
\hline Valladolid provincia & 12 & $27 \%$ \\
\hline - $\quad$ Otra provincia & 1 de Palencia & $2 \%$ \\
\hline \multicolumn{3}{|l|}{ MOMENTO DE INICIO: } \\
\hline - $\quad$ Congénita & 30 & $67 \%$ \\
\hline - $\quad$ Tardía: & 13 & $29 \%$ \\
\hline $\begin{array}{l}\text { Prelocutiva ( } 0 \text { a } 2 \text { años) } \\
\text { (\% de adquiridas/\%de totales) }\end{array}$ & 7 & $(54 \% / 16 \%)$ \\
\hline Perilocutiva (2 a 4 años) & 4 & $(31 \% / 9 \%)$ \\
\hline Postlocutiva (> 4 años) & 2 & $(15 \% / 9 \%)$ \\
\hline - $\quad$ De inicio desconocido & 2 & $4 \%$ \\
\hline \multicolumn{3}{|l|}{ TIPO DE HIPOACUSIA: } \\
\hline • $\quad$ Neurosensorial & 39 & $87 \%$ \\
\hline Transmisión & 4 & $9 \%$ \\
\hline • $\quad$ Mixta & 2 & $4 \%$ \\
\hline \multicolumn{3}{|l|}{ GRADO DE HIPOACUSIA: } \\
\hline - $\quad$ Leve $(30-40 \mathrm{~dB})$ & 8 & $18 \%$ \\
\hline Moderada (41-70 dB) & 12 & $27 \%$ \\
\hline Severa $(71-90 \mathrm{~dB})$ & 10 & $22 \%$ \\
\hline - $\quad$ Profunda $(>90 \mathrm{~dB})$ & 15 & $33 \%$ \\
\hline \multicolumn{3}{|l|}{ HIPOACUSIAS ASOCIADAS: } \\
\hline - $\quad$ No asociadas & 28 & $62 \%$ \\
\hline Asociadas otras altcs. no sindrómicas & 12 & $27 \%$ \\
\hline - $\quad$ Asociadas a Síndromes & 5 & $11 \%$ \\
\hline \multicolumn{3}{|l|}{ PATOLOGÍA OÍDO MEDIO: } \\
\hline - $\quad$ No padecimientos & 16 & $36 \%$ \\
\hline OMA recurrente u OMS crónica & 29 & $64 \%$ \\
\hline - $\quad$ Colocación de drenajes transtimpánicos & 14 & $31 \%$ \\
\hline \multicolumn{3}{|l|}{ ADAPTACIÓN PROTÉSICA: } \\
\hline - $\quad$ Sin adaptación & 6 & $13 \%$ \\
\hline Audífonos bilaterales & 22 & $49 \%$ \\
\hline • Implante coclear & 17 & $38 \%$ \\
\hline \multicolumn{3}{|l|}{ TIPO DE IMPLANTACIÓN (IC): $(\mathrm{N}=17)$ : } \\
\hline • Unilateral & 7 & $(41 \% / 15 \%)$ \\
\hline • $\quad$ Bilateral secuencial & 5 & $(29,5 \% / 11 \%)$ \\
\hline - $\quad$ Bilateral simultáneo & 5 & $(29,5 \% / 11 \%)$ \\
\hline
\end{tabular}

Desde el punto de vista auditivo, las características de la población hipoacúsica escogida, se muestran en la Tabla 2. Destaca el porcentaje de varones sobre hembras, como suele ser habitual en el reparto de la hipoacusia infantil. Al menos un $15 \%$ presenta retraso mental, que junto a las alteraciones del comportamiento y los déficits específicos del lenguaje 
(no evaluados inicialmente) van a ser un factor determinante en el pronóstico evolutivo. Casi la mitad proceden del cribado auditivo realizado en el hospital, por lo que son pacientes que conocemos desde su nacimiento. Dos tercios de las hipoacusias son congénitas y todas ellas diagnosticadas en el primer año de vida (el $80 \%$ con menos de 6 meses). La mayoría de las hipoacusias son neurosensoriales y aproximadamente la mitad de grado severo a profundo. A su vez, todas las de grado severo a profundo son exclusivamente neurosensoriales. Un tercio de los pacientes asocia otra patología, incluyendo la sindrómica en 5 casos ( 1 Down, 1 CHARGE, 1 Waardenburg y 2 Usher, que son hermanos). Dos tercios de los niños estudiados sufrieron patología crónica o recurrente de oído medio, la mitad de los cuales precisó la colocación de drenajes transtimpánicos. La hipoacusia derivada de esta patología, que se añade a la existente y las dificultades que en ocasiones genera con los medios de amplificación, hacen que deba ser considerada como un factor agravante [19]. Podemos decir que tan solo 8 niños no incluyen ninguna excepción de entre los criterios de inclusión y tampoco han presentado otras alteraciones ni patología de oído medio. Finalmente, la adaptación protésica se realizó en todos los pacientes (salvo en algunos casos de hipoacusia leve a moderada). La mitad de los audífonos se adecuaron en el primer año de edad y casi todos los IC se realizaron antes de cumplir los 2 años.

Conocidas las características auditivas de los casos, queremos saber qué otras variables podrían influir en el desarrollo general y en el del lenguaje en particular, por ser el motivo principal de este estudio. Para ello elaboramos una entrevista, que deben contestar los padres y/o cuidadores (Anexo 1) donde hemos intentado incluir todos aquellos factores que la literatura y nosotros, en función del contexto en el que nos movemos, consideramos de interés. La hemos dividido en varios apartados: edad y ciclo escolar, vivienda, características socioeconómicas de la unidad familiar, intervención, participación familiar y otros. En términos generales, se recoge información relativa al medio escolar y logopédico del niño, de sus impresiones respecto a la adquisición de las distintas competencias lingüísticas, tipos y usos de adaptación protésica y otras formas de amplificación, modos complementarios de comunicación (lectura labial, signos), afectación auditiva de los padres, desórdenes de lenguaje en la familia, grado de educación del cuidador principal y finalmente, ámbito familiar que rodea al niño. Para determinar la participación familiar, utilizaremos The Moeller's Family Rating Scale [20], que caracteriza en una escala de 1 a 5 el grado de implicación de la familia (Anexo 1).

A la vez que se completa esta entrevista con los padres y cuidadores y con los informes logopédicos y psicopedagógicos que nos aportan, comenzamos la aplicación de las pruebas de lenguaje a los niños. Las seleccionadas en función de la edad (Tabla 3), son las siguientes:

- La discriminación auditiva se valora mediante el EDAF (Discriminación Auditiva y Fonológica), que consta de 5 subtests: discriminación de sonidos del medio, discriminación figura/fondo, discriminación fonológica en palabras, discriminación fonológica en logotomas y memoria secuencial auditiva.

- La evaluación fonética y fonológica, se realizará de 3 a 6 años mediante el RFI (Registro Fonológico Inducido) y a partir de esta edad se aplicará el Registro Fonológico de Laura Bosch.

- El PLON-R (Prueba de Lenguaje Oral de Navarra, Revisado) será aplicado a los 3 y 4 años para obtener información de diferentes aspectos del lenguaje oral. En concreto, nosotros recogeremos el pragmático y el morfosintáctico. A partir de los 5 años, esta información será registrada por medio del BLOC-SR (Batería de Lenguaje Objetiva y Criterial, Screening).

- Evaluaremos el lenguaje comprensivo mediante el PPVT-III (Test de Vocabulario en Imágenes PEABODY) y el CEG (Comprensión de Estructuras Gramaticales). Y el expresivo mediante la de WISC-IV (Escala de Vocabulario).

- Finalmente, a partir de los 7 años, aplicaremos el EMLE (Escalas Magallanes de Lectura y Escritura) para evaluar las dificultades en la lectoescritura. Comprende varios subtests: lectura en voz alta, comprensión lectora, copia y dictado.

Estas pruebas se administrarán siguiendo un orden determinado:

- Las primeras serán el BLOC-SR o el PLON$R$, en función de la edad. Si existieran dificultades a nivel semántico, se pasarán las pruebas correspondientes de WISC-IV y 
PEABODY. Si en el BLOC-SR se obtuviera una edad equivalente a la cronológica, no se realizarían las pruebas de nivel semántico.
- Posteriormente se procederá con las pruebas de fonología y finalmente con las de lectoescritura.

Tabla 3. Pruebas de lenguaje utilizadas en función de la edad.

\begin{tabular}{|c|c|c|c|c|c|c|c|}
\hline \multirow{2}{*}{$\begin{array}{c}\text { AÑO DE } \\
\text { NACIMIENTO }\end{array}$} & \multirow{2}{*}{$\begin{array}{l}\text { DISCRIMINACIÓN } \\
\text { AUDITIVA } \\
\end{array}$} & \multirow{2}{*}{$\begin{array}{c}\text { FONÉTICO } \\
\text { FONOLÓGICO } \\
\end{array}$} & \multirow{2}{*}{ MORFOSINTÁCTICO } & \multicolumn{2}{|c|}{ SEMÁNTICO } & \multirow[t]{2}{*}{ PRAGMÁTICO } & \multirow{2}{*}{$\begin{array}{l}\text { LECTURA Y } \\
\text { ESCRITURA }\end{array}$} \\
\hline & & & & COMPRENSIVO & EXPRESIVO & & \\
\hline 2013 & EDAF & RFI & PLON-R & PEABODY & WISC & PLON - R & - \\
\hline 2012 & EDAF & RFI & PLON - R & PEABODY & WISC & PLON-R & - \\
\hline 2011 & EDAF & RFI & PLON-R & PEABODY & WISC & PLON-R & - \\
\hline 2010 & EDAF & RFI & BLOC-SR & PEABODY & WISC & BLOC-SR & - \\
\hline 2009 & EDAF & RFI & BLOC-SR & PEABODY & WISC & BLOC - SR & - \\
\hline 2008 & EDAF & RFLB & BLOC-SR & PEABODY & WISC & BLOC - SR & EMLE \\
\hline 2007 & EDAF & RFLB & BLOC-SR & PEABODY & WISC & BLOC - SR & EMLE \\
\hline 2006 & EDAF & RFLB & BLOC-SR & PEABODY & WISC & BLOC-SR & EMLE \\
\hline 2005 & EDAF & RFLB & BLOC-SR & PEABODY & WISC & BLOC-SR & EMLE \\
\hline 2004 & EDAF & RFLB & BLOC-SR & PEABODY & WISC & BLOC-SR & EMLE \\
\hline 2003 & EDAF & RFLB & BLOC-SR & PEABODY & WISC & BLOC-SR & EMLE \\
\hline 2002 & EDAF & RFLB & BLOC-SR & PEABODY & WISC & BLOC - SR & EMLE \\
\hline 2001 & EDAF & RFLB & BLOC - SR & PEABODY & WISC & BLOC - SR & EMLE \\
\hline
\end{tabular}

A fin de que la terminología y comparación de resultados pueda resultar más sencilla, obtendremos de cada prueba la edad equivalente de cada uno de los niveles evaluados. Puesto que los estándares más habituales son las puntuaciones centiles, clasificaremos los resultados finales de cada caso en función de las mismas, en 7 rangos:

- RANGO 1 (puntuación muy baja): centil por debajo de 10.

- RANGO 2 (puntuación baja): centil entre 10 y 25.

- RANGO 3 (puntuación normal baja): centil de 25 a 35.

- RANGO 4 (puntuación normal): centil entre 35 y 65.

- RANGO 5 (puntuación normal alta): centil de 65 a 75 .

- RANGO 6 (puntuación alta): centil entre 75 y 90.

- RANGO 7 (puntuación muy alta): centil por encima de 90.

Para facilitar la evaluación de resultados desde el punto de vista estadístico, procuraremos realizar grupos de casos lo más uniformes posibles. A partir de los 7 rangos definidos en los resultados de la exploración del lenguaje obtendremos 3 grandes grupos:

- GRUPO 1: BUENA EVOLUCIÓN: que se corresponde a los RANGOS 3 a 7, ambos inclusive.

- GRUPO 2: EVOLUCIÓN REGULAR: corresponde al RANGO 2.
- gRUPO 3: MALA EVOLUCIÓN: comprende a los casos del RANGO 1.

Dentro de cada grupo tendremos a un conjunto de niños cuyas características podremos analizar estadísticamente y compararlas con el resto, permitiéndonos conocer cuáles son las más relevantes e influyentes en el pronóstico.

\section{DISCUSIÓN}

Parece demostrado que el diagnóstico e intervención precoz de la hipoacusia neonatal permiten alcanzar unos niveles de lenguaje similares a los de los niños normoyentes [8-10], pero es impredecible saber quiénes lo lograrán. Realizar estudios prospectivos sobre el lenguaje en niños y niñas con hipoacusia es difícil, porque muchos factores pueden afectar su progreso. Entre los publicados, destacamos las actualizaciones realizadas por Ching et al. (2013) [21] y por Fulcher et al. (2015) [22] donde se refiere que los mejores resultados en relación a las características del niño y de su familia se asocian con: género femenino, elevado cociente intelectual, buen nivel socioeconómico y educativo de la madre, correcta pronunciación materna y uso de técnicas facilitadoras del lenguaje, pocos hermanos, ausencia de otras discapacidades, participación en los servicios logopédicos, uso continuado de audífonos, existencia de restos auditivos antes de la implantación coclear e hipoacusia adquirida en vez de congénita. Para los niños con hipoacusia severa a profunda que reciben implante coclear, el mayor 
rendimiento se obtiene con la adaptación precoz de la amplificación, la implantación coclear temprana (antes de los 18 meses de edad) y bilateral, de la más moderna tecnología. Los niveles de lenguaje también mejoran si la intervención pretende desarrollar habilidades auditivoverbales frente a un lenguaje signado. $Y$ es preferible un centro escolar común a otro especializado [21].

Tobei et al. (2003) [23] sintetizan en 3 elementos el conjunto de variables determinantes. El desarrollo del habla y del lenguaje en el niño con hipoacusia neonatal va a depender de las peculiaridades del niño y de su familia, de las características de la amplificación y de la forma inicial de habilitación (centrada en comunicación oral, signada o mixta). Para Sarant et al. (2009) [24], la participación familiar, el grado de hipoacusia y la capacidad cognitiva fueron los principales predictores de los niveles de lenguaje obtenidos. Yoshinaga-Itano et al. (1998) [25], opinan que la edad de identificación/intervención y la capacidad cognitiva son los elementos determinantes. Para Moeller (2000) [20], aplicando modelos de regresión múltiple, los dos factores fundamentales serían la edad de inicio de la intervención y la implicación familiar. En definitiva, al ser tantos los componentes definidos, no existe acuerdo para decidir cuáles son los más importantes en determinar el mejor desarrollo del lenguaje.

De este conjunto de factores, hay unos que pueden modificarse (como la edad de identificación y de implantación coclear, tipo de intervención o el modo de comunicación) y otros no (como el tipo y grado de hipoacusia, nivel socioeconómico y educativo de la familia). Tanto unos como otros influyen en el resultado final del habla y del lenguaje del niño, por lo que de su conocimiento es posible que podamos adelantar un pronóstico individualizado y facilitar un desarrollo más dirigido [26]. Por tal motivo, hemos recopilado los datos que la literatura contempla como variables y una vez estructurados en diferentes apartados, hemos confeccionado la entrevista que llevaremos a cabo.

Para hacerse una idea de la amplitud y variabilidad de estudios publicados sobre el desarrollo del lenguaje en el niño/a con hipoacusia, Thompson et al., (2001) [27], nos presenta un resumen de los trabajos iniciales hasta el año 2000 y Pimperton y Kennedy (2012) [28], los actualizan hasta el 2010, donde se incluye el impacto del cribado auditivo universal en el desarrollo del habla y del lenguaje. Además de los realizados por su grupo y el de McCann
[29] en Inglaterra, destacan los de EEUU [25, 30], Canadá [31], Australia [24, 32] y en Europa además de los mencionados, los de Holanda [33], Suecia [34], Italia [35], etc., no habiendo encontrado referencias de trabajos similares en nuestro país.

En relación a los descritos, las características diferenciales de este estudio son las siguientes:

- La edad de evaluación en otros trabajos, suele ser fija y muy temprana (3, 5 o 7 años) [20, 25, 30-35], por lo que el rango al que nosotros llegamos (de 3 a 15 años) podría mostrarnos diferencias evolutivas.

- Aunque en la metodología de la mayor parte de estudios, incluido el nuestro, se mezcla el registro de variables a través de un cuestionario y el estudio del lenguaje del niño/a, hay quien sólo emplea el interrogatorio a los padres, incluso a través de correo, para conocer el nivel lingüístico del niño/a y no es infrecuente que de realizarse la evaluación, sólo se base en el vocabulario receptivo o en la inteligibilidad, sin analizar otros aspectos [32]. En este trabajo, no sólo hemos incluido las principales variables que pueden influir en el grado de desarrollo, sino que la valoración del lenguaje comprende todos sus elementos formales, al considerar que la deficiencia auditiva puede afectarlos de manera diferente, en función de la evolución.

- Finalmente, una gran cantidad de investigaciones llevadas a cabo sobre el progreso del habla y del lenguaje en niños y niñas con hipoacusia, se han centrado en la hipoacusia profunda, cuando la mayoría de los afectados/as, la tienen leve, moderada o severa (y los que la tienen severa/profunda son candidatos a prótesis o IC y tras su colocación se transforman en hipoacusias leves/moderadas) [34]. En nuestro estudio, hemos contemplado todos los grados de hipoacusia, de leve a profundo.

\section{LIMITACIONES DEL ESTUDIO}

Cuando se intenta resolver un problema con tantas variables, se precisan muchos participantes para obtener sólidas conclusiones, porque además el estudio debe realizarse en una población que presente una cierta uniformidad. Pero dada la baja prevalencia de la hipoacusia neonatal (3\% recién nacidos) [2], resulta también difícil comparar los resultados, pues los publicados muestran diversos grupos de edad, 
examinados en épocas diferentes, con características desiguales, y con pruebas y herramientas distintas [24]. El número de niños seleccionados en este trabajo, aunque por los datos de prevalencia y los criterios establecidos representan un grupo importante, son una población escasa desde el punto de vista estadístico, lo que en algún caso puede dificultar la obtención de una suficiente significación, pero pretendemos revelar los factores más representativos de la evolución del lenguaje del niño con hipoacusia y contrastarlos con los obtenidos por otros autores.

Si por una parte, la aplicación de los criterios de selección permite uniformizar el grupo de estudio, por otra, limita los resultados a las circunstancias establecidas, dejando para el futuro la evaluación de otras variables como: mayores de 15 años, retraso psíquico añadido, hipoacusias progresivas, unilaterales, etc.

Al organizar y distribuir los casos analizados entre cuatro grupos de trabajo, podrían aparecer diferencias en los resultados de la evaluación. Este inconveniente se ha intentado paliar de tres maneras. En primer lugar, realizando un ensayo clínico previo, donde se estableció un protocolo de pautas de actuación y comportamiento, comunes para todos los participantes. En segundo lugar, distribuyendo de manera uniforme la población a estudiar por edades entre los 4 grupos, y finalmente, utilizando el registro de las grabaciones audiovisuales de las exploraciones fundamentales, para un posible examen posterior por todos los colaboradores, con el fin de discutir los criterios de homogeneidad que pudieran faltar.

Como cabe la posibilidad de que alguna de las pruebas de lenguaje seleccionadas se haya pasado recientemente por un profesional externo, se deberá decidir en estos casos, si se repite la prueba o se tienen en cuenta los resultados previamente obtenidos, en función exclusivamente de la prueba realizada y del periodo transcurrido, con independencia de que su realización sea ajena a este estudio.

Tras aplicar durante 15 años el cribado universal y la intervención precoz de la hipoacusia permanente neonatal en el HCU-VA, las circunstancias nos han permitido llevar a cabo la evaluación del lenguaje en estos niños. La consonancia de medios ofrece un preciso control de los resultados, mejorando la calidad de los datos obtenidos. La derivación de pacientes nos ha proporcionado una amplia pobla- ción, que hubiera supuesto el resultado de cribar a 94.000 recién nacidos durante más de 60 años. Finalmente, la oportunidad de coincidir especialistas de la audición y del lenguaje, ha sido otro elemento decisivo para este trabajo. Aunque como hemos explicado, la variabilidad y escasez de población son factores limitantes, confiamos en que otros favorezcan la posibilidad de obtener resultados válidos.

\section{CONCLUSIONES}

Desde el punto de vista clínico y asistencial, conocer el desarrollo del lenguaje adquirido por los niños y niñas con hipoacusia prelingual, diagnosticados precozmente en un hospital de la red sanitaria pública y siguiendo un proceso de intervención estandarizado, significa validar la metodología utilizada en nuestro medio para el diagnóstico y tratamiento de la hipoacusia infantil. Pero como los resultados dependen de las características de la población, este estudio también documentará el desarrollo del lenguaje de los niños con hipoacusia de nuestro entorno.

\section{AGRADECIMIENTOS}

A todos los profesionales, personal de enfermería y compañeros que con su trabajo y consejos hicieron posible que el cribado auditivo y la intervención precoz de la hipoacusia neonatal se llevaran a cabo de la mejor forma posible y a todos los niños y niñas que han participado en este proyecto cuyas familias aceptaron ofrecernos su apoyo incondicional.

\section{BIBLIOGRAFÍA}

1. JCIH (2007). Joint Committee on Infant Hearing: Principles and guidelines for early hearing detection and intervention programs (Position Statement). Pediatrics. 2007;120:898-921.

2. Benito-Orejas JI, Ramírez B, Morais D, Fernández-Calvo JL, Almaraz $\mathrm{A}$. Resultados de aplicar durante 42 meses un protocolo universal de detección e intervención precoz de la hipoacusia en neonatos. Acta Otorrinolaringol Esp.2008;59(3):96-101.

3. Fitzpatrick E, Angus D, Durieux-Smith A, Graham ID, Coyle D. Parent's needs following identification of childhood hearing loss. Am J Audiol 2008;17(1): 38-49. 
4. Vohr B, Jodoin-Krauzyk J, Tucker R, Johnson MJ, Topol D, Ahlgren M. Early language outcomes of early-identified infants with permanent hearing loss at 12 to 16 months of age. Pediatrics. 2008;122(3):535-44

5. Mohr PE, Feldman JJ, Dunbar JL, McConkey-Robbins A, Niparko JK, Rittenhouse RK, et al. The societal costs of severe to profound hearing loss in the United States. Int $\mathrm{J}$ Technol Assess Health Care. 2000;16(4):1120-35.

6. Schroeder L, Petrou S, Kennedy C, McCann D, Law C, Watkin PM, et al. The economic costs of congenital bilateral permanent childhood hearing impairment. Pediatrics. 2006;117(4):1101-12.

7. Watkin PM. Neonatal hearing screening: methods and outcome. Audiol Med. 2003;1:165-74.

8. Geers AE, Moog JS, Biedenstein J, Brenner $\mathrm{C}$, Hayes $\mathrm{H}$. Spoken language scores of children using cochlear implants compared to hearing age-mates at school entry. J Deaf Stud Deaf Educ. 2009;14(3):371-85.

9. Verhaert N, Willems M, Van Kerschaver E, Desloovere C. Impact of early hearing screening and treatment on language development and education level: evaluation of 6 years of universal newborn hearing screening (ALGO) in Flanders, Belgium. Int J Pediatr Otorhinolaryngol. 2008;72(5):599-608.

10. Yoshinaga-Itano C, Baca RL, Sedey AL. Describing the trajectory of language development in the presence of severe-to-profound hearing loss: a closer look at children with cochlear implants versus hearing aids. Otol Neurotol. 2010;31(8):1268-74.

11. Keren R, Helfand M, Homer C, McPhillips $H$, Lieu TA. Projected cost-effectiveness of statewide universal newborn hearing screening. Pediatrics. 2002;110(5):855-64.

12. Isaacson G. Universal newborn hearing screening in an inner-city, managed care environment. Laryngoscope. 2000;110:881-94.

13. Comisión para la detección precoz de la hipoacusia infantil (CODEPEH). Propuesta para la detección e intervención precoz de la hipoacusia infantil. An Esp Pediatr.1999;51:336-344.
14. Martínez R, Benito Jl, Condado MA, Morais $\mathrm{D}$, Fernández-Calvo JL. Resultados de aplicar durante 1 año un protocolo universal de detección precoz de la hipoacusia en neonatos. An Otorrinolaringol Ibero Am. 2003;30(3):277-87.

15. Junta de Castilla y León. Consejería de Sanidad. Programa de detección precoz y atención integral de la hipoacusia infantil. 2004. 78 pp.

16. Benito-Orejas JI, Ramírez B, Morais D, Almaraz A, Fernández-Calvo JL. Comparison of two-step transient evoked otoacoustic emissions (TEOAE) and automated auditory brainstem response (AABR) for universal newborn hearing screening programs. Int J Pediatr Otorhinolaryngol. 2008;72(8):1193-201.

17. Benito-Orejas JI, Pardal-Refoyo JL. Evidencia y recomendación: ¿Cuál es la mejor técnica para el cribado auditivo neonatal?. Rev ORL. 2016;7(2):97-102. DOI: http://dx.doi.org/10.14201/orl201672.14680.

18. Benito-Orejas JI, Poncela-Blanco M, García-Vicario F, Benito-González F, Martín-Sigüenza G, San Román-Carbajo J. ¿Es fácil encargarse de coordinar un «programa de hipoacusia infantil»? Rev ORL. 2016;7(2):7790.

DOI: http://dx.doi.org/10.14201/orl201672.142 37.

19. Boudewyns A, Declau F, Van den Ende J, Van Kerschaver E, Dirckx S, Hofkens-Van den Brandt $A$, et al. Otitis media with effusion: an underestimated cause of hearing loss in infants. Otol Neurotol. 2011;32(5):799-804.

20. Moeller MP. Early intervention and language development in children who are deaf and hard of hearing. Pediatrics. 2000;106(3):E43.

21. Ching TY, Dillon $H$, Marnane V, Hou S, Day $\mathrm{J}$, Seeto $\mathrm{M}$, et al. Outcomes of early- and lateidentified children at 3 years of age: findings from a prospective population-based study. Ear Hear. 2013;34(5):535-52.

22. Fulcher AN, Purcell A, Baker E, Munro N. Factors influencing speech and language outcomes of children with early identified severe/profound hearing loss: Clinician-identified facilitators and barriers. Int J Speech Lang Pathol. 2015;17(3):325-33. 
23. Tobey EA, Geers AE, Brenner C, Altuna D, Gabbert G. Factors associated with development of speech production skills in children implanted by age five. Ear Hear. 2003;24(1 Suppl):36S-45S.

24. Sarant JZ, Holt CM, Dowell RC, Rickards FW, Blamey PJ. Spoken language development in oral preschool children with permanent childhood deafness. J Deaf Stud Deaf Educ. 2009;14(2):205-17.

25. Yoshinaga-Itano C, Sedey AL, Coulter DK, Mehl AL. Language of early- and later-identified children with hearing loss. Pediatrics. 1998;102(5):1161-71.

26. Fulcher A, Purcell AA, Baker E, Munro N. Listen up: children with early identified hearing loss achieve age-appropriate speech/language outcomes by 3 years-of-age. Int J Pediatr Otorhinolaryngol. 2012;76(12):1785-94.

27. Thompson DC, McPhillips H, Davis RL, Lieu TL, Homer CJ, Helfand M. Universal newborn hearing screening: summary of evidence. JAMA. 2001;286(16):2000-10.

28. Pimperton $\mathrm{H}$, Kennedy $\mathrm{CR}$. The impact of early identification of permanent childhood hearing impairment on speech and language outcomes. Arch Dis Child. 2012;97(7):648-53.

29. McCann DC, Worsfold S, Law CM, Mullee M, Petrou S, Stevenson J, et al. Reading and communication skills after universal newborn screening for permanent childhood hearing impairmentArch Dis Child. 2009;94(4):293-7.
30. Yoshinaga-Itano C, Coulter D, Thomson V. The Colorado Newborn Hearing Screening Project: effects on speech and language development for children with hearing loss. J Perinatol. 2000;20(8 Pt 2):S132-7.

31. Fitzpatrick E, Durieux-Smith A, Eriks-Brophy A, Olds J, Gaines R. The impact of newborn hearing screening on communication development. J Med Screen. 2007;14(3):123-31.

32. Wake M, Poulakis Z, Hughes EK, CareySargeant C, Rickards FW. Hearing impairment: a population study of age at diagnosis, severity, and language outcomes at $7-8$ years. Arch Dis Child. 2005;90(3):238-44.

33. Korver AM, Konings S, Dekker FW, Beers $\mathrm{M}$, Wever CC, Frijns JH, et al. DECIBEL Collaborative Study Group. Newborn hearing screening vs later hearing screening and developmental outcomes in children with permanent childhood hearing impairment. JAMA. 2010;304(15):1701-8.

34. Borg E, Edquist G, Reinholdson AC, Risberg A, McAllister B. Speech and language development in a population of Swedish hearing-impaired pre-school children, a cross-sectional study. Int J Pediatr Otorhinolaryngol. 2007;71(7):1061-77.

35. Bubbico L, Di Castelbianco FB, Tangucci $M$, Salvinelli $F$. Early hearing detection and intervention in children with prelingual deafness, effects on language development. Minerva Pediatr. 2007;59(4):307-13. 
DESARROLLO DEL LENGUAJE EN NIÑOS CON DETECCIÓN TEMPRANA DE LA HIPOACUSIA NEONATAL BENITO-OREJAS JI ET AL.

Anexo 1

Nombre del investigador/a:

№ participante:

\section{ENTREVISTA INICIAL}

FECHA DE ENTREVISTA:

Sexo: Masculino / Femenino

Fecha de nacimiento:

Edad actual (en años y meses):

Rechaza participación: No / Si

Pérdida de seguimiento: No / Si

Datos incompletos o no válidos:

\begin{tabular}{|l|c|c|}
\hline EDAD Y CICLO ESCOLAR: \\
\hline Educación Infantil & Primer ciclo (0-3 años) & Segundo ciclo (3-6 años) \\
\hline Educación Primaria & Primero (6-7 años) & Segundo (7-8 años) \\
\cline { 2 - 3 } & Tercero (8-9 años) & Cuarto (9-10 años) \\
\cline { 2 - 3 } & Quinto (10-11 años) & Sexto (11-12 años) \\
\hline \multirow{2}{*}{ Educación Secundaria Obligatoria } & Primero (12-13 años) & Segundo (13-14 años) \\
\cline { 2 - 4 } & Tercero (14-15 años) & Cuarto (15-16 años) \\
\hline
\end{tabular}

VIVIENDA:

Localidad: $\quad$ Valladolid ciudad

\begin{tabular}{|l|l|l|}
\hline \multirow{2}{*}{} & Valladolid (zona rural) & $\begin{array}{l}\text { Km hasta servicios médicos, } \\
\text { escolares... más cercanos: }\end{array}$ \\
\cline { 2 - 3 } & Otros & \\
\hline $\begin{array}{l}\text { Tipo de vivienda (piso, } \\
\text { chalé, adosado, otro) } \\
\text { (propiedad o alquiler): }\end{array}$ & & \\
\hline $\begin{array}{l}\text { No de personas que } \\
\text { conviven: }\end{array}$ & \\
\hline $\begin{array}{l}\text { Persona con la que más } \\
\text { tiempo pasa el sujeto: }\end{array}$ & \\
\hline Apoyos en el domicilio: & \\
\hline $\begin{array}{l}\text { Ayudas técnicas en el } \\
\text { domicilio: }\end{array}$ & \\
\end{tabular}

Página 1 de 7 
DESARROLLO DEL LENGUAJE EN NIÑOS CON DETECCIÓN TEMPRANA DE LA HIPOACUSIA NEONATAL BENITO-OREJAS JI ET AL.

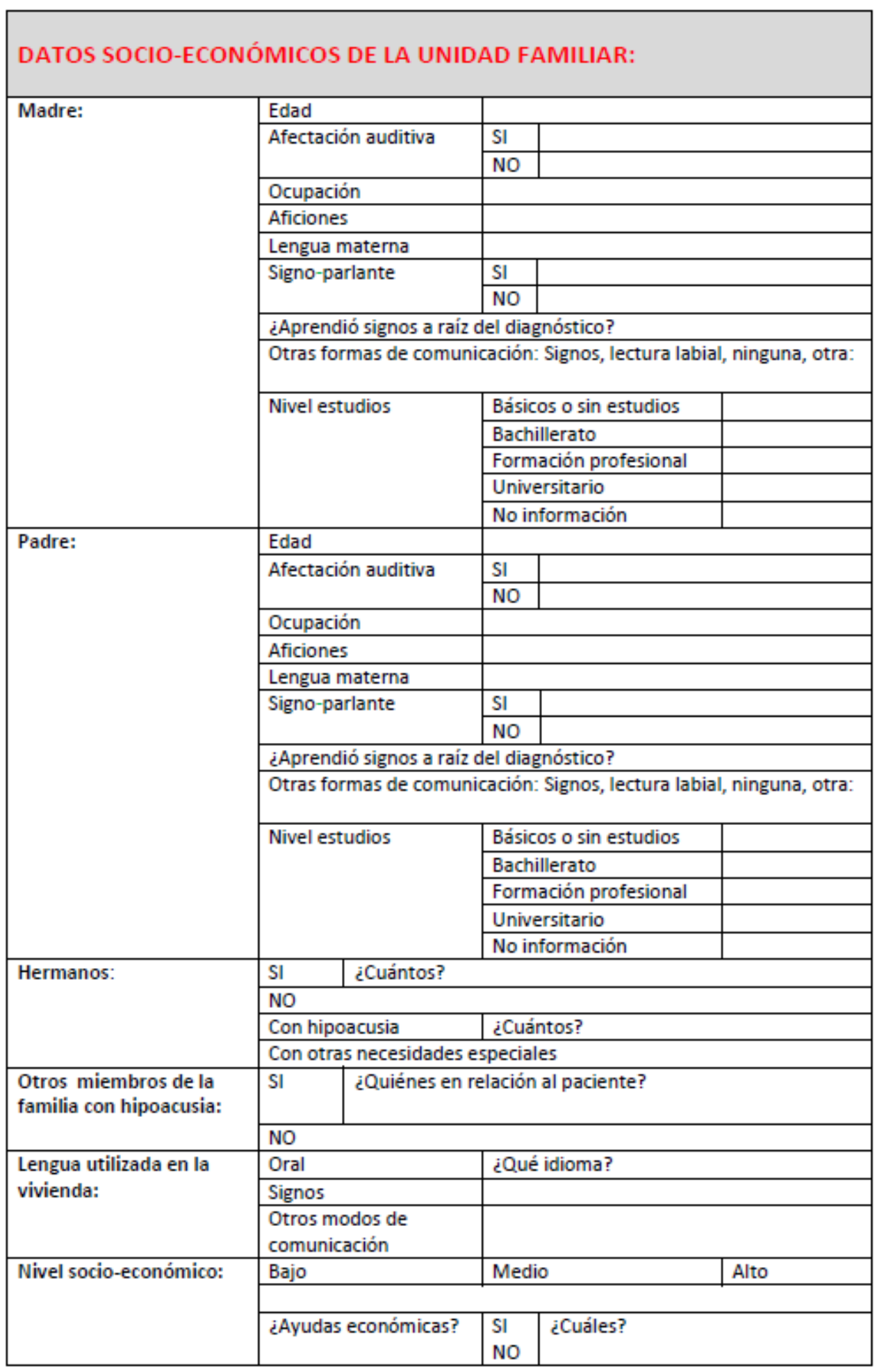

Página 2 de 7 
DESARROLLO DEL LENGUAJE EN NIÑOS CON DETECCIÓN TEMPRANA DE LA HIPOACUSIA NEONATAL BENITO-OREJAS JI ET AL.

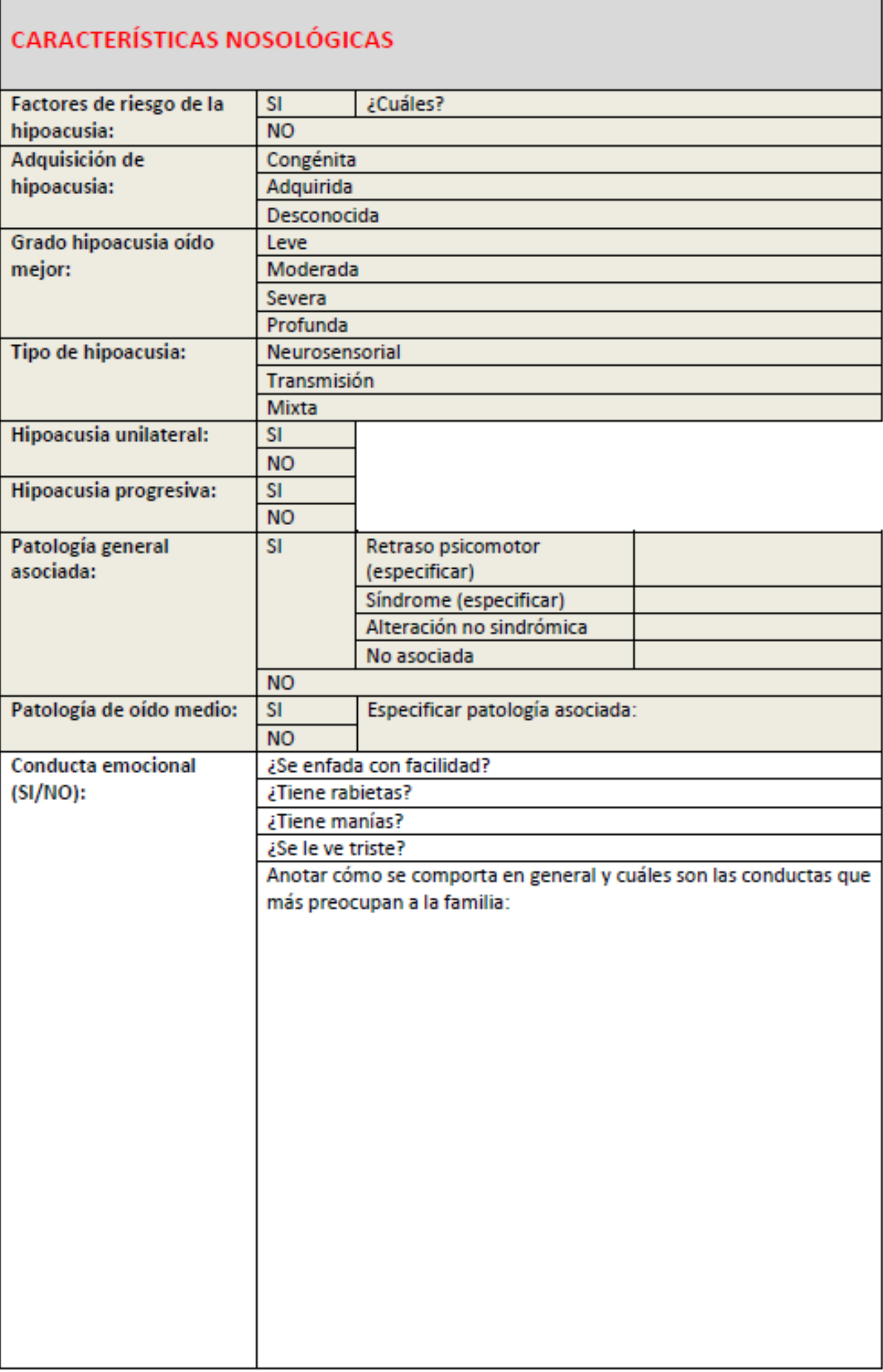

Página 3 de 7 


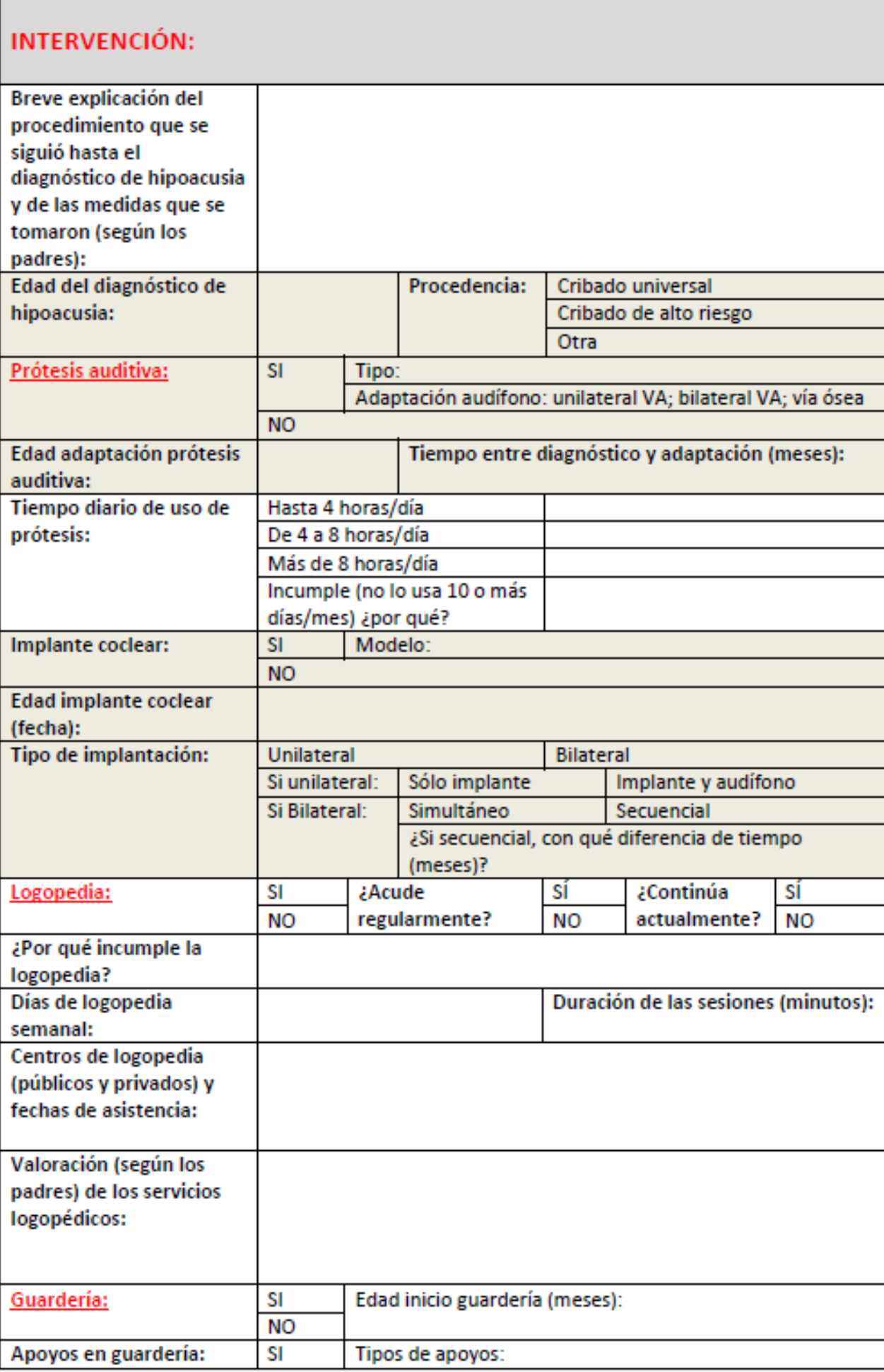

Página 4 de 7 
DESARROLLO DEL LENGUAJE EN NIÑOS CON DETECCIÓN TEMPRANA DE LA HIPOACUSIA NEONATAL BENITO-OREJAS JI ET AL.

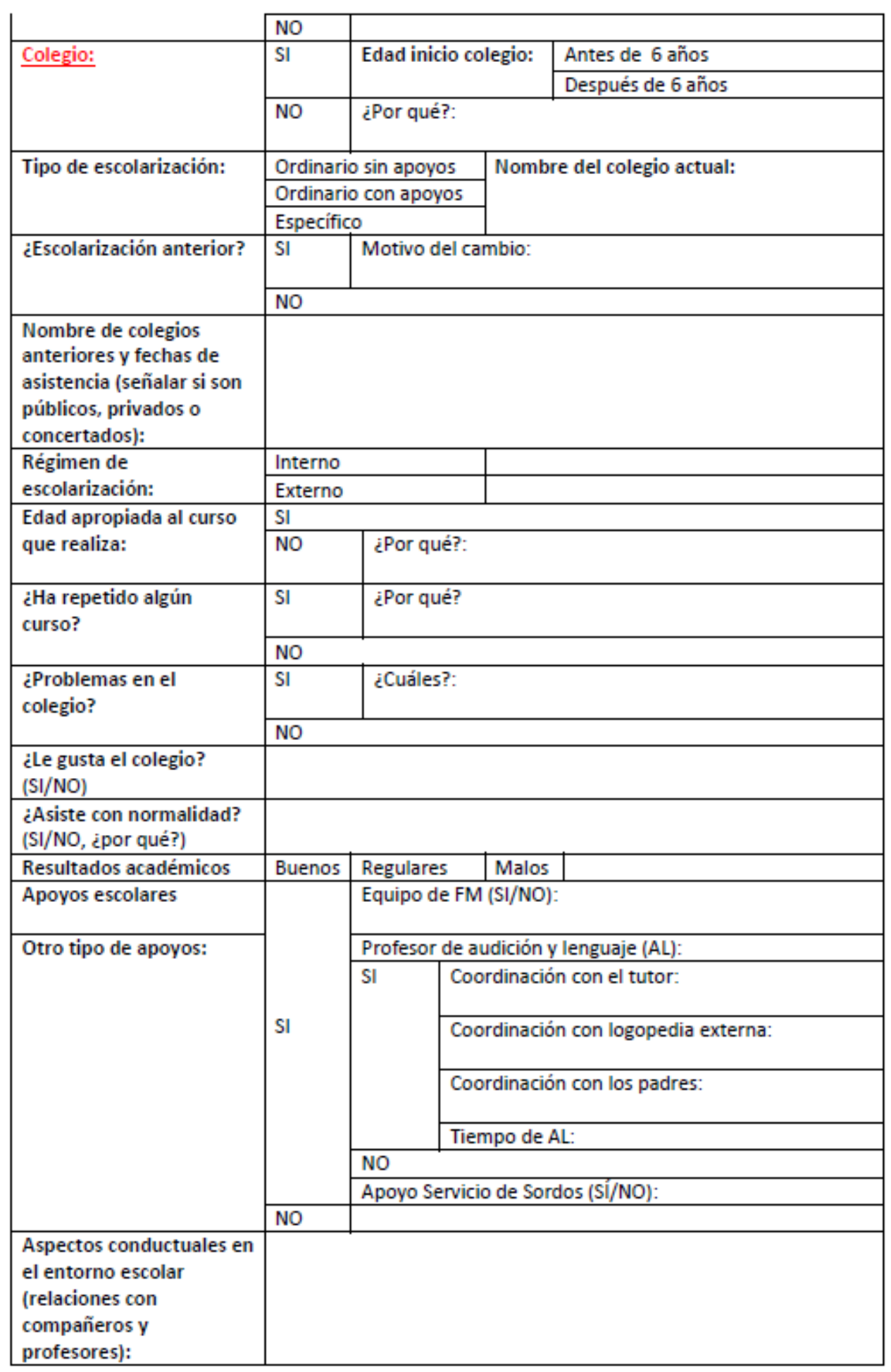

Página 5 de 7 
DESARROLLO DEL LENGUAJE EN NIÑOS CON DETECCIÓN TEMPRANA DE LA HIPOACUSIA NEONATAL BENITO-OREJAS JI ET AL.

\begin{tabular}{|c|c|c|}
\hline $\begin{array}{l}\text { Observaciones: (nivel de } \\
\text { satisfacción de los padres } \\
\text { sobre la calidad de los } \\
\text { servicios escolares) }\end{array}$ & & \\
\hline PARTICIPACIÓN FAM & ILIAF & \\
\hline ¿Cómo aceptan la & Con & nalidad \\
\hline sordera de su hijo? & Luch & or aceptarla \\
\hline & Nol & tienden \\
\hline ¿Cómo ven el desarrollo & Nor & \\
\hline de su hijo? & $\begin{array}{l}\text { Retr } \\
\text { ¿por }\end{array}$ & \\
\hline $\begin{array}{l}\text { ¿Centra la discapacidad } \\
\text { como tarea familiar? }\end{array}$ & SI & Medidas: \\
\hline & NO & \\
\hline $\begin{array}{l}\text { ¿Quiénes de la familia } \\
\text { participan con el niño? }\end{array}$ & & \\
\hline $\begin{array}{l}\text { ¿Acuden a las sesiones de } \\
\text { logopedia? }\end{array}$ & SI & ¿Quiénes? \\
\hline & NO & \\
\hline ¿Acuden a conferencias & SI & \\
\hline sobre hipoacusia? & NO & \\
\hline ¿Buscan información en & $\mathrm{SI}$ & \\
\hline el entorno? & NO & \\
\hline ¿Participan en los & $\mathrm{SI}$ & \\
\hline $\begin{array}{l}\text { patrones de lenguaje } \\
\text { establecidos? }\end{array}$ & NO & \\
\hline $\begin{array}{l}\text { ¿Cómo afrontan el } \\
\text { trastorno de su hijo?: }\end{array}$ & & \\
\hline
\end{tabular}

Página 6 de 7 
DESARROLLO DEL LENGUAJE EN NIÑOS CON DETECCIÓN TEMPRANA DE LA HIPOACUSIA NEONATAL BENITO-OREJAS JI ET AL.

Moeller MP: Early Intervention Efficacy Project: Escala de Participación Familiar.

\begin{tabular}{|c|c|}
\hline 5 (participación ideal): & $\begin{array}{l}\text { La familia ha aceptado plenamente la sordera de su hijo y centra esta discapacidad como una } \\
\text { tarea familiar. Los miembros familiares se comprometen activamente en las sesiones. Acuden } \\
\text { regularmente a las sesiones, a laz conferencias y buscan información de su entorno. Se } \\
\text { muestran partíipes de lo que se les offece con los profesionales o escuelas. Los miembros } \\
\text { familiares participan con efectividad en los patrones de lenguaje establecidos con el nin̄o, con } \\
\text { quien se comunican permanentemente, utilizando de forma efectiva y fluente el modo de } \\
\text { comunicación del niño. Son capaces de aplicar técnicaz que expandan la comprensión del } \\
\text { lenguaje. El resto de los miembros familiares también están involucrados y participan. }\end{array}$ \\
\hline 4 (buena participación): & $\begin{array}{l}\text { La familia ha aceptado, mejor que la media, la sordera de su hijo. Los miembros familiares } \\
\text { acuden regularmente a conferenciza y sesiones. Los padres tiejnen un papel activo (pero no } \\
\text { son líderes) en los planes de logopedia y educación. Participan en los patrones de lenguaje } \\
\text { establecidos y se esfuerzan en trasladarlos a su casa. Algunos miembros familiares tienen } \\
\text { facilidad para comunicarse con el niño en el modo establecido y en técnicas de estimulación } \\
\text { del lenguaje. Se effuerzan para que se involucre el resto de la familila. }\end{array}$ \\
\hline $\begin{array}{l}3 \text { (forma mayoritaria, } \\
\text { promedio, de } \\
\text { participación): }\end{array}$ & 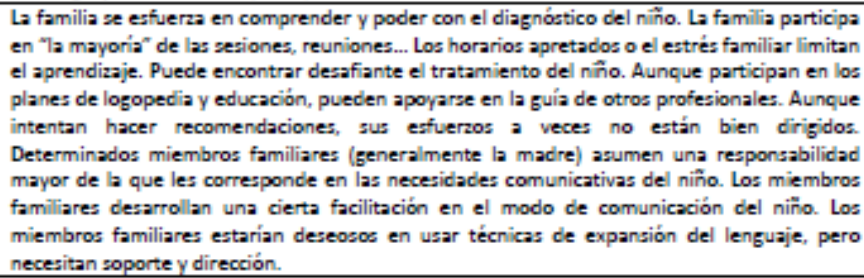 \\
\hline $\begin{array}{l}2 \text { (por debajo de la } \\
\text { media): }\end{array}$ & $\begin{array}{l}\text { La familia lucha para aceptar el diagnóstico del niña. La atención familiar es inconsistente. } \\
\text { Son inconstantes en el mantenimiento de los audífonos en perfectas condiciones dentro y } \\
\text { fuera de la escuela. Pueden tener determinados problemas que interfieren con el consistente } \\
\text { trasada de aprendizaje a casa. El tratamiento del niño genera problemas cotidianos en la } \\
\text { familia. La interacción comunicativa con el niño es bázica. A loa familia le falta facilidad en el } \\
\text { modo de comunicación del niño. }\end{array}$ \\
\hline 1 (escasa participación): & $\begin{array}{l}\text { La familia tiene significativos problemas que son más importantes que las necesidades del } \\
\text { niño (abuso doméstico, falta de vivienda... La familia comprende poco el significado de la } \\
\text { sordera y sus consecuencias. La participación es esporádica e inefectiva. La comunicación de } \\
\text { los padres con el nin̂́o se limita a las necesidades básicas. }\end{array}$ \\
\hline \multicolumn{2}{|l|}{ OTROS ASPECTOS: } \\
\hline \multicolumn{2}{|c|}{$\begin{array}{l}\text { Valoración general de los padres hacia los servicios de salud, escolares... y hacia el } \\
\text { tratamiento/s médicos, logopédicos, quirúrgicos, escolares... }\end{array}$} \\
\hline \multicolumn{2}{|c|}{ ¿Quiere hacernos algún comentario o solicitud?: } \\
\hline \multicolumn{2}{|l|}{ Observaciones: } \\
\hline
\end{tabular}

Página 7 de 7 